\title{
Identificación de serogrupos de Leptospira spp en alpacas del Centro de Investigación IVITA (Maranganí, Cusco)
}

\author{
IDENTIFICATION of Leptospira SPp SEROGROUPS IN ALPACAS FROM THE IVITA RESEARCH \\ Centre (Marangani, Cusco)
}

Yaquelyn Better B. ${ }^{1}$, Guillermo Salvatierra ${ }^{1}$, Juan Siuce M. ${ }^{1}$, Sonia Calle E. ${ }^{1,2}$

\section{Resumen}

El objetivo del estudio fue identificar los serogrupos de Leptospira spp presentes en alpacas de la estación experimental Maranganí del Centro de Investigación IVITA, Cusco, Perú. Se recolectaron 126 muestras de sangre de alpacas mayores a un año y se analizaron mediante la prueba serológica de microaglutinación (MAT), utilizando serovares de referencia internacional de 21 serogrupos patógenos. Se determinó que el 42.9\% (34.5$51.7 \%$ ) de las alpacas fueron seropositivas a nueve serogrupos patógenos: Icterohaemorrhagiae (12.7\%), Pomona (11.1\%), Panama (11.1\%), Hurstbridge (9.5\%), Ranarum (5.6\%), Ballum (1.6\%), Cypnopteri (1.6), Djasiman (0.8\%) y Hebdomadis (0.8\%). Asimismo, se obtuvo el 2.4\% (3/126) de coaglutinaciones.

Palabras clave: Leptospira; serogrupos; microaglutinación; alpacas

\section{Abstract}

The aim of this study was to identify the Leptospira spp serogroups present in alpacas from the Maranganí Experimental Station of the IVITAResearch Center in Cusco, Peru. Blood samples from 126 alpacas older than one year of age were collected and analyzed by the serological microagglutination test (MAT). Serovars of international reference of 21 pathogenic serogroups were used. Results showed that $42.9 \%$ (34.51$51.7 \%$ ) of alpacas were seropositive to nine pathogenic serogroups: Icterohaemorrhagiae (12.7\%), Pomona (11.1\%), Panama (11.1\%), Hurstbridge (9.5\%), Ranarum (5.6\%), Ballum (1.6\%), Cypnopteri (1.6), Djasiman (0.8\%) and Hebdomadis (0.8\%). Likewise, $2.4 \%(3 / 126)$ of coagglutinations were obtained.

Key words: Leptospira; serogroups; microagglutination; alpaca

\footnotetext{
${ }^{1}$ Laboratorio de Microbiología y Parasitología Veterinaria, Facultad de Medicina Veterinaria, Universidad Nacional Mayor de San Marcos, Lima, Perú

${ }^{2}$ E-mail: scallee@unmsm.edu.pe
}

Recibido: 7 de agosto de 2017

Aceptado para publicación: 27 de marzo de 2018 


\section{INTRODUCCIÓN}

La leptospirosis es una enfermedad zoonótica bacteriana de distribución mundial, causada por espiroquetas patógenas del género Leptospira que infectan a humanos y mamíferos, principalmente a aquellos que habitan regiones tropicales y subtropicales (Levett, 2001; Adler y De la Peña, 2015). No obstante, en las áreas altoandinas donde las condiciones medioambientales son desfavorables para la supervivencia de las leptospiras se han reportado casos en humanos, así como una alta prevalencia en camélidos sudamericanos (Céspedes, 2005; Rosadio et al., 2012).

La infección ha sido asociada en la alpaca a cuadros de abortos y nacimiento de crías débiles (Ellis, 2015), afectando negativamente su crianza y rendimiento económico (Herrera et al., 2000). En un estudio realizado en el altiplano puneño se obtuvo $6.5 \%$ de reacción seropositiva, siendo el serovar Pomona el de mayor frecuencia (Herrera et al., 2000). En la zona colindante a la provincia de Canchis, Cusco, se determinó mayor prevalencia de los serovares Pomona, Icterohaemorragiae y Canicola (Rosadio et al., 2003). Por otro lado, en 66 comunidades campesinas de los departamentos de la sierra central (Huancavelica, Ayacucho) se determinó que el $89.6 \%$ de las alpacas reaccionaron seropositivamente a cuatro serovares patógenos, especialmente a Icterohaemorrhagiae y Pomona (Rosadio et al., 2012).

La leptospirosis es, además, una enfermedad asociada a la actividad laboral. Los trabajadores se exponen a suelos y agua contaminados con orina de animales infectados, como es el caso de los ganaderos, veterinarios y trabajadores de mataderos, entre otros (Levett, 2001).

La identificación de serogrupos en una especie animal permite identificar su rol potencial en el mantenimiento y diseminación de leptospiras patógenas, intermedias o saprófitas a otras especies y al medio am- biente. Además, es determinante para la elección del panel de antígenos en la prueba diagnóstica de microaglutinación, a fin de incrementar la sensibilidad de la prueba y disminuir los costos de su realización (Picardeau, 2013). Un panel incompleto podría ocasionar falsos negativos y la no identificación de los serogrupos presentes en una especie o región (Musso y La Scola, 2013). Por ello, el objetivo del presente estudio fue identificar los serogrupos de Leptospira spp en un rebaño de alpacas de Puno, Perú, utilizando serovares representativos de 21 serogrupos patógenos en la prueba de microaglutinación.

\section{Materiales y Métodos}

\section{Localización}

La toma de muestras se realizó en marzo de 2014 en el rebaño de alpacas de la Estación Experimental Maranganí del Centro de Investigación IVITA, ubicada en la provincia de Canchis, Cusco. El desarrollo de la prueba serológica se realizó en el Laboratorio de Bacteriología de la Facultad de Medicina Veterinaria, Universidad Nacional Mayor de San Marcos, en Lima.

\section{Animales}

Se recolectaron muestras de sangre (3 $\mathrm{ml})$ de 126 alpacas mayores de un año, escogidas al azar en los hatos de machos y hembras. Los animales se encontraban en aparente buen estado de salud y no registraban brotes de abortos. Las muestras fueron extraídas por punción de la vena yugular (Fowler, 2010). Los sueros se obtuvieron por centrifugación y se almacenaron a $-20^{\circ} \mathrm{C}$.

\section{Prueba de Microaglutinación}

La detección de anticuerpos específicos contra los serogrupos de Leptospira spp se realizó mediante la prueba de microaglutinación (MAT), prueba de referencia internacional para el diagnóstico de 
leptospirosis humana y animal (OIE, 2008). Se seleccionaron serovares representativos de 21 serogrupos patógenos: Australis, Autumnalis, Ballum, Bataviae, Canicola, Celledoni, Cynopteri, Djasiman, Grippotyphosa, Hebdomadis, Hurstbridge, Icterohaemorrhagiae, Louisiana, Manhao, Mini, Panama, Pomona, Pyrogenes, Ranarum, Sejroe y Shermani. Para la selección de los serovares se siguieron los criterios de la guía para el diagnóstico, vigilancia y control de la OMS (2008).

Se realizó una prueba tamiz para identificar las muestras seropositivas y sospechosas $(\geq 1 / 100)$. En estas muestras se determinaron la seropositividades en diluciones hasta 1/1600. La reacción de aglutinación se visualizó en el microscopio de campo oscuro, observando movimiento de los extremos libres de las leptospiras. El punto de corte o título final utilizado fue el definido por el Subcomité de Taxonomía en Leptospira (1984) como la dilución de suero $(\geq 1 / 100)$ que muestre $50 \%$ de aglutinación y deje $50 \%$ de leptospiras libres en relación al antígeno control. Las muestras seropositivas a dos o más serogrupos con títulos semejantes fueron consideradas como coaglutinaciones.

\section{Análisis Estadístico}

Los resultados por serogrupos fueron expresados como frecuencias con sus respectivos intervalos de confianza del $95 \%$. Se utilizó el programa estadístico R-project para realizar la simulación en la distribución beta y calcular el límite superior e inferior del intervalo de confianza para 30000 observaciones.

\section{Resultados y Discusión}

El 42.9\% ( $\mathrm{n}=126)$ de las alpacas fueron seropositivas a nueve serogrupos patógenos de Leptospira: Icterohaemorrhagiae, Pomona, Panama, Hurstbridge, Ranarum, Ballum, Cypnopteri, Djasiman y
Hebdomadis (Cuadro 1). Los títulos bajos fueron los de mayor frecuencia, mientras que un reducido número de muestras presentaron títulos altos (Cuadro 2). El 2.4\% de las muestras presentaron coaglutinaciones a serogrupos patógenos (Cuadro 3).

La frecuencia se seropositividad de este estudio fue menor al $6.5 \%$ registrado por Herrera et al. (2000) en Puno y e inferior al 89.6\% encontrado por Rosadio et al. (2012) en las comunidades de Ayacucho y Huancavelica. No obstante, fue similar al $44.8 \%$ reportado por Santos et al. (2009) en alpacas del Instituto Nacional de Innovación Agraria (INIA) de Quimsachata, Puno.

La distribución beta indicó diferencias significativas $(p<0.05)$ entre las frecuencias de los serogrupos Icterohaemorrhagiae, Pomona y Panana con los serogrupos Ballum, Cynopteri, Hebdomadis y Djasiman. Las mayores frecuencias de seropositivos correspondieron a los serogrupos Pomona (11.1\%) e Icterohaemorrhagiae $(12.7 \%)$ con títulos máximos de $1 / 1600$ y $1 / 200$, respectivamente. Las frecuencias de los serogrupos Pomona e Icterohaemorrhagiae fueron menores que las reportadas por Rosadio et al. (2012) con 37.8 y 43.4\%, respectivamente, en las zonas de Huancavelica y Ayacucho y muy superiores a las reportadas por Herrera et al. (2000) de 5.2 y $0.25 \%$ para Pomona e Icterohaemorrhagiae en la zona de Puno. Cabe resaltar que estos serogrupos han sido los de mayor frecuencia en los estudios de prevalencia realizados en las alpacas y otras especies de camélidos sudamericanos (Santos et al., 2009; Rosadio et al., 2012).

La baja frecuencia de títulos altos indicaría una infección reciente o enfermedad aguda si se presentasen signos clínicos, mientras que títulos bajos podrían estar asociados a exposiciones anteriores $\mathrm{o}$ al historial de una infección, esto es si se toma en consideración que estas alpacas no cuentan con un calendario de vacunación contra la leptospirosis. Asimismo, si se considera que 
Cuadro 1. Frecuencia de serogrupos patógenos de Leptospira spp en alpacas $(\mathrm{n}=126)$ de la Estación Experimental Maranganí del Centro de Investigación IVITA (Cusco, Perú, 2014)

\begin{tabular}{lccc}
\hline Serogrupos & $\begin{array}{c}\text { Muestras } \\
\text { seropositivas }\end{array}$ & $\begin{array}{c}\text { Frecuencia } \\
(\%)\end{array}$ & $\mathrm{IC}_{(0.95) \%}$ \\
\hline Icterohaemorrhagiae & 16 & 12.7 & $8.0-19.6$ \\
Pomona & 14 & 11.1 & $6.8-17.8$ \\
Panama & 14 & 11.1 & $6.8-17.8$ \\
Hurstbridge & 12 & 9.5 & $4.2-12.2$ \\
Ranarum & 7 & 5.6 & $2.8-11.0$ \\
Ballum & 2 & 1.6 & $0.5-5.5$ \\
Cynopteri & 2 & 1.6 & $0.5-5.5$ \\
Djasiman & 1 & 0.8 & $0.2-4.3$ \\
Hebdomadis & 1 & 0.8 & $0.2-4.3$ \\
\hline
\end{tabular}

Cuadro 2. Títulos de anticuerpos contra serogrupos patógenos de Leptospira spp de alpacas seropositivas $(\mathrm{n}=54)$ de la Estación Experimental Maranganí del Centro de Investigación IVITA (Cusco, Perú, 2014)

\begin{tabular}{lccccc}
\hline \multirow{2}{*}{ Serogrupos } & \multicolumn{5}{c}{ Títulos } \\
\cline { 2 - 6 } & $1 / 100$ & $1 / 200$ & $1 / 400$ & $1 / 800$ & $1 / 1600$ \\
\hline Ballum & 2 & - & - & - & - \\
Cynopteri & 2 & - & - & - & - \\
Djasiman & 1 & - & - & - & - \\
Hebdomadis & 1 & - & - & - & - \\
Hurstbridge & 1 & 5 & 1 & 3 & 2 \\
Icterohaemorrhagiae & 12 & 4 & - & - & - \\
Panama & 2 & 9 & 3 & - & - \\
Pomona & 1 & 10 & 2 & - & 1 \\
Ranarum & 3 & 3 & 1 & - & - \\
\hline
\end{tabular}

un hospedero de mantenimiento puede ser seronegativo a la prueba de MAT, como es el caso del ganado bovino infectado por el serovar Hardjo, un resultado negativo o un título por debajo del punto de corte estableci- do $(1 / 100)$ en esta prueba no excluiría una infección crónica en las alpacas, ni el diagnóstico de aborto o la posibilidad de ser identificado como un hospedero de mantenimiento (OIE, 2008). 
Pese a que las condiciones medioambientales de las zonas altoandinas son adversas para la supervivencia de las leptospiras, la infección de las alpacas por el serogrupo Pomona podría estar asociado al tipo de sistema de producción, en el que se aprovecha los pastos naturales y cultivados mediante el pastoreo mixto de diferentes especies domésticas como los bovinos, ovinos y camélidos (Herrera et al., 2000; Rosadio et al., 2003). Este serogrupo tiene como hospedero de mantenimiento a los cerdos y se caracteriza por producir infecciones esporádicas en el ganado bovino y equino ocasionando fallas reproductivas, infertilidad, abortos, mortalidad perinatal, nacimiento de crías débiles y disminución de la producción láctea (Grooms y Bolin, 2005; Ellis, 2015). En las alpacas y demás especies de camélidos, las leptospiras han sido consideradas como causa de abortos a pesar de que existen pocos reportes relevantes sobre el rol de estos organismos en la presentación de abortos en estas especies (Pearson et al., 2014).

En los camélidos sudamericanos, el serogrupo Icterohaemorrhagiae no ha sido vinculado a algún cuadro clínico específico; mientras que en humanos y perros ocasiona graves cuadros clínicos ictero-hemorrágicos, insuficiencia hepática-renal e incluso la muerte (Céspedes, 2005). En el periodo 1994-2004 se encontró la presencia del serogrupo Icterohaemorrhagiae en 15 departamentos del Perú, incluyendo Cusco y Huancavelica, lugares donde han sido reportados camélidos sudamericanos seropositivos a este serogrupo por la prueba de MAT (Céspedes et al., 2006). La diseminación de este serogrupo en la mayor parte del territorio nacional es multifactorial y estaría favorecida, además, por el nicho ecológico y la bacteriuria continúa de su principal reservorio: los roedores domésticos y sinantrópicos (Levett, 2001).

El presente estudio señala que existen anticuerpos contra los serogrupos patógenos Ballum, Djasiman, Hurtbridge, Panama y Ranarum en las alpacas del IVITA Maran- ganí. Estos serogrupos no habían sido reportados en alpacas, posiblemente debido al panel incompleto de serogrupos patógenos evaluado en los estudios previos. Los serogrupos Ballum y Djasiman han sido identificados en casos humanos de 13 departamentos del Perú, mientras que el serogrupo Panama ha sido únicamente reportado en los departamentos de Ayacucho y Loreto (Céspedes et al., 2006).

Se obtuvo $2.38 \%$ (3/126) de coaglutinaciones, principalmente con los serogrupos patógenos Hurstbridge y Pomona, con títulos de $1 / 200$, lo cual estaría indicando la coexistencia de una infección subaguda anterior o crónica a determinado serogrupo y una infección actual a diferente serogrupo (AndreFontaine, 2006).

\section{Conclusiones}

- Las alpacas de la Estación Experimental Maranganí del Centro de Investigación IVITA resultaron seropositivas contra nueve serogrupos patógenos de Leptospira: Icterohaemorrhagiae, Pomona, Hurstbridge, Panama, Ranarum, Ballum, Cypnopteri, Djasiman y Hebdomadis.

- Los serogrupos patógenos Ballum, Djasiman, Hurstbridge, Panama y Ranarum no habían sido reportados anteriormente en alpacas en el Perú.

\section{Literatura Citada}

1. Adler B, De la Peña M. 2010. Leptospira. En: Gyles CL, Prescott JF, Songer G, Thoen CO (eds). Pathogenesis of bacterial infections in animals. $4^{\text {th }} \mathrm{ed}$. USA: Wiley Blackwell. p 527-547.

2. André-Fontaine G. 2006. Canine leptospirosis - Do we have a problem? Vet Microbiol 117: 19-24. doi: 10.1016/ j.vetmic.2006.04.005 
3. Céspedes ZM. 2005. Leptospirosis: enfermedad zoonótica reemergente. Rev Perú Med Exp Salud Pública 22: 290-307.

4. Céspedes ZM, Balda L, Gonzalez D, Tapia R. 2006. Situación de la leptospirosis en el Perú 1994-2004. Rev Peru Med Exp Salud Publica 23: 56-66.

5. Ellis WA. 2015. Animal leptospirosis. In: Adler B (ed). Leptospira and leptospirosis. Current topics in microbiology and immunology. Berlín: Springer. p 98-137.

6. Fowler ME. 2010. Medicine and surgery of camelids. $3^{\text {rd }}$ ed. Singapore: Wiley Blackwell. $630 \mathrm{p}$.

7. Groom DL, Bolin CA. 2005. Diagnosis of fetal loss caused by bovine viral diarrhea virus and Leptospira spp. Vet Clin North Am Food Anim Pract 21: 463472. doi: 10.1016/j.cvfa.2005.02.010

8. Herrera $J$, Vasconcellos $S$, Morais $Z$, Ferreira F, Sakamoto S, Ferreira J, Pinheiro S. 2000. Soropositividade para leptospirose em alpacas criadas no altiplano peruano. Puno, Peru. Análise de associação com o índice pluviometrico. Arq Inst Biol 67: 171-176.

9. International Committee on Systematic Bacteriology, Subcommittee on the Taxonomy of Leptospira. 1984. Minutes of the meeting, 6 to 10 August 1982, Boston, Massachusetts. Int J Syst Bacteriol 34: 258-259.

10. Levett PN. 2001. Leptospirosis. Clin Microbiol Rev 14: 296-326. doi: 10.1128/ CMR.14.2.296-326.2001

11. Musso D, La Scola B. 2013. Laboratory diagnosis of leptospirosis: a challenge. J Microbiol Immunol Infect 46: 245-252. doi: 10.1016/j.jmii.2013.03.001

12. [OIE] Organización Mundial de la Sanidad Animal. 2008. Manual de las pruebas diagnósticas y de vacunas para los animales terrestres. Cap. 2.1.9.
Leptospirosis. [Internet]. Disponible en: http://web.oie.int/esp/normes/mmanual/ pdf_es_2008/2.01.09.\%20 Leptospirosis.pdf

13. [OMS] Organización Mundial de la Salud. 2008. Leptospirosis humana: Guía para el diagnóstico, vigilancia y control. Serie de manuales técnicos $\mathrm{N}^{\mathrm{o}} 12$. [Internet]. Disponible en: http:// www.med.monash.edu.au/microbiology/ staff/adler/guia-esp.pdf

14. Pearson LK, Rodriguez JS, Tibary A. 2014. Disorders and diseases of pregnancy. In: Cebra C, Anderson DE, Tibary A, et al. (eds). Llama and alpaca care: medicine, surgery, reproduction, nutrition and herd health. Canada: Saunders Elservier. p 256- 273.

15. Picardeau M. 2013. Diagnosis and epidemiology of leptospirosis. Méd Mal Infect 43: 1-9. doi: 10.1016/ j.medmal.2012.11.00

16. Rosadio R, Rivera H, Chávez A, Serrano E, Quispe R, Rodríguez J, Yaya $K$. 2003. Seroprevalencia de agentes abortigénicos en alpacas de la provincia de Canchis (Cusco, Perú). En: III Congreso Mundial de Camélidos. Oruro, Bolivia.

17. Rosadio R, Véliz A, Castillo H, Yaya $K$, Rodríguez A, Rivera H, Wheeler J. 2012. Seroprevalencia a serovares de Leptospiras patógenas en alpacas y vicuñas de los departamentos de Huancavelica y Ayacucho. Perú Rev Inv Vet Perú 23: 350-356. doi: 10.15381/ rivep.v23i3.916

18. Santos Y, Suárez F, Rivera H, Huanca W, Cárdenas O, Camacho J. 2009. Seroprevalencia de leptospirosis en alpacas de Quimsachata, Puno. Rev Inv Vet Perú 20: 108-113. doi: 10.15381/ rivep.v20i1.559 